OPEN ACCESS

UNIVERSITY OF THE

WEST of SCOTLAND

UWS Academic Portal

\title{
Interval-based parameters for stress diffusion in granular medium
}

Boumezerane, Djamalddine

Published in:

Safety and Reliability - Safe Societies in a Changing World

DOI:

$10.1201 / 9781351174664$

Published: 15/06/2018

Document Version

Publisher's PDF, also known as Version of record

Link to publication on the UWS Academic Portal

Citation for published version (APA):

Boumezerane, D. (2018). Interval-based parameters for stress diffusion in granular medium. In S. Haugen, A. Barros, C. van Gulijk, T. Kongsvik, \& J. E. Vinnem (Eds.), Safety and Reliability - Safe Societies in a Changing World: Proceedings of ESREL 2018, June 17-21, 2018, Trondheim, Norway (pp. 2621-2626). Taylor \& Francis. https://doi.org/10.1201/9781351174664

\section{General rights}

Copyright and moral rights for the publications made accessible in the UWS Academic Portal are retained by the authors and/or other copyright owners and it is a condition of accessing publications that users recognise and abide by the legal requirements associated with these rights.

Take down policy

If you believe that this document breaches copyright please contact pure@uws.ac.uk providing details, and we will remove access to the work immediately and investigate your claim. 


\title{
Interval-based parameters for stress diffusion in granular medium
}

\author{
D. Boumezerane \\ Middle East Technical University, Northern Cyprus, METU \\ Formerly Norwegian University of Science and Technology, NTNU, Norway
}

\begin{abstract}
According to Bourdeau (1986), diffusion of stresses in a granular medium can be described using a probabilistic approach. A point load applied on the surface of a granular media will follow an erratic path, depending on the probability of transition between the grains. The diffusion of the expected vertical stress in the granular medium can be described by a Fokker-Planck type equation. In terms of expected vertical stresses, an equation of diffusion is obtained and the parameter of diffusion is shown to approximate the coefficient of lateral pressure of the material at a given depth $\mathrm{z}$. The coefficient of lateral pressure of the material can be expressed in terms of intervals with upper and lower values to account for uncertainty.

In the present approach, we propose to solve the diffusion equation using interval-based parameters to account for uncertainty. Uncertain parameters are considered as discretized fuzzy numbers; they are combined with finite difference method to solve the diffusion equation. Comparisons are made with experimental and available data.
\end{abstract}

\section{INTRODUCTION}

Diffusion of stresses in granular media is a phenomenon that provokes rearrangement in grains and thus settlements. Soils as granular media are constituted of an assembly of particles of different sizes, mineralogy and morphologies. The particle sizes vary from less than $0.002 \mathrm{~mm}$ in clays to some tens of millimeters in gravel materials. Despite their granular aspect, they are considered, from the soil mechanics viewpoint, as a continuum. Theories of continuous media are often applied to model the behavior of cohesive materials such as clays. Cohesionless soils behavior on the other hand is difficult to capture using continuum approaches. Their granular aspect, especially when they are in loose states, makes their behavior complex to predict using conventional theories of continuum media.

Harr (1977) proposed an approach to estimate the expected vertical stress in a granular medium subjected to surficial loading. A concentrated load applied on the surface of a semi-infinite ground will follow an uncertain path between the grains. The resulting stress in one point is a random variable. Its distribution will reflect the composition of the media. Following a binomial law of diffusion on one side or the other given a reference mark, the transmission of the load in the granular medium will be expressed as an equation of diffusion of stresses. The main parameter characterizing the equation is the coefficient of lateral pressure in soils. Bourdeau (1986) extended this approach and proposed a formulation in terms of displacements diffusion in loose cohensionless granular medium.

We revisited the formulation in terms of stress diffusion to account for parameter uncertainty using intervals which can be combined and expressed also as fuzzy discretized numbers. A numerical approach based on finite difference method is combined with interval-based parameters to simulate diffusion of stresses in granular media. Interval-based parameters are used to account for uncertainty and comparisons are made with experimental data. The aim of this research is to study parameter uncertainty and its quantification in the context of stress diffusion in a granular medium.

\section{DIFFUSION OF STRESSES IN A GRANULAR MEDIUM}

Diffusion of stresses in soils is a fundamental aspect of soil mechanics. It is observed in all soilstructure interaction problems as a consequence of applied loads. Harr (1977) build a model for stress diffusion in a granular medium based on the idea of expected stress distribution at a point. A unit force applied on the surface will follow a random path between the grains. The vertical normal stress acting at a point within a medium is the total accumulated effect of many random variables; shape and distribution of particles, the spatial distribution of voids as well as their local configurations. 
The central limit theorem assures that the distribution of stress will converge to the normal distribution as the number of particles becomes large (Harr, 1977).

In Figure 1 is shown, schematically, the transmission of vertical forces between particles. The effect of the boundary force will spread laterally in the positive and negative directions. At a representative particle, the input stress can be taken to divide in the left and right directions consistent with a Bernoulli trial. The division of stresses is expected to be equal. For a random distribution the frequency of moving to the left is the same as moving to the right. Figure 2 shows the distribution of stresses within a homogeneous random medium. If $\Delta x$ is the average spacing of the stresses at row, with the rows taken to be $\Delta z$ apart, the expected stress will follow a binomial distribution with the recurrence equations (Harr, 1977):

$$
\bar{S}_{z}[x, z+\Delta z]=\frac{1}{2}\left\{\bar{S}_{z}[x-\Delta x, z]+\bar{S}_{z}[x+\Delta x, z]\right\}
$$

Subtracting the expected vertical stress $\bar{S}_{z}[x, z]$ from each side of the expression and dividing by $\Delta z$, we get:

$$
\begin{aligned}
\frac{\bar{S}_{z}[x, z+\Delta z]-\bar{S}_{z}[x, z]}{\Delta z}=\frac{(\Delta x)^{2}}{2 \Delta z} \\
\left\{\frac{\bar{S}_{z}[x+\Delta x, z]-2 \bar{S}_{z}[x, z]+\bar{S}_{z}[x-\Delta x, z]}{(\Delta x)^{2}}\right\}
\end{aligned}
$$

In the limit as $\Delta x$ and $\Delta z$ become very small, the equation can be expressed as a differential one:

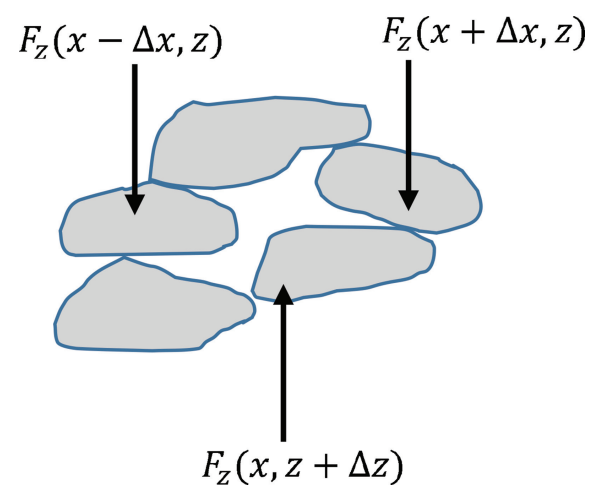

Figure 1. Transmission of vertical forces.

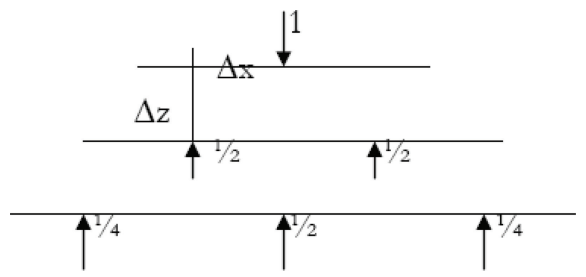

Figure 2. Unit stress distribution in terms of probabilities.

$\frac{\partial \bar{S}_{z}}{\partial z}=D \frac{\partial^{2} \bar{S}_{z}}{\partial x^{2}}$

where $D=\lim _{\Delta x, \Delta z \rightarrow 0}\left\{\frac{1}{2} \frac{\Delta x^{2}}{\Delta z}\right\}$

The ratio $\frac{\Delta x^{2}}{\Delta z}$ is seen to reflect a characteristic of the particulate medium. Harr (1977) has shown that $(D=v . z)$ is dependent on the depth $z$ and the coefficient of lateral stress $v$. The author also showed that $v$ can be approximated by the coefficient of earth pressure at rest $K$ (using Jaky's formula for example).

Equation (3) is an equation of diffusion of expected vertical stresses in a granular media, with $D$ the parameter of characterization.

The main objective of our research is to study the variability of the coefficient $D$ and its influence on the diffusion of stresses in a granular medium. Uncertainty analysis will be carried out based on interval analysis and fuzzy representations of $D$. Different sources of information are used to consider the coefficient of diffusion.

\subsection{Uncertainty in the diffusion coefficient}

Uncertainties in geotechnics are generally classified into two categories, "epistemic" related to lack of data or knowledge and "aleatory" related to natural randomness (Baecher \& Christian, 2003). The diffusion parameter $\mathrm{D}$ is subjected to epistemic uncertainties which have influence on the diffusion process in the granular medium. The main sources of uncertainty of D come from evaluation of lateral pressure coefficient $K$ as $=K . z$. In situ and laboratory measures are used to evaluate lateral pressure coefficient in soils via empirical relations (Jaky's formula for example). Empirical correlations combining Jaky's formula $\left(1-\sin \varphi^{\prime}\right)$ with OCR from laboratory are also used to determine the lateral pressure coefficient at rest $K_{0}$ (Cai et al. 2011). When experimental results are given in terms of intervals the average values are generally used.

Figure 3 shows variation of lateral earth pressure based on different tests (Chen \& Fang, 2008). 


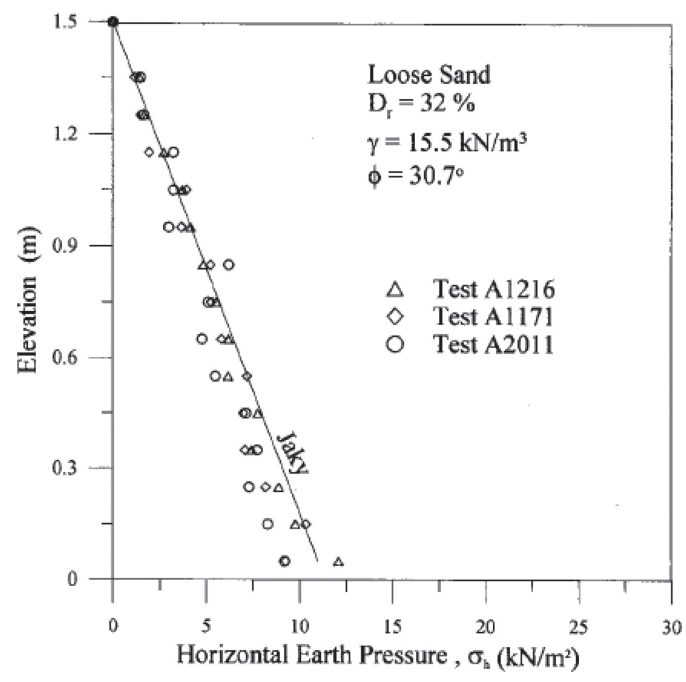

Figure 3. Distribution of horizontal earth pressure against model wall (Cheng \& Fang, 2008).

Chen \& Fang (2008) studied variation of lateral earth pressure in a block of sand. They presented experimental data on the variation of lateral earth pressure against a non-yielding retaining wall due to soil filling and vibratory compaction.

Schmertmann et al. (2005) used a K-Box device to describe stress diffusion in sand under a surface circular plate loading. The experiment intended to improve understanding of stress distribution in a particulate medium. Comparisons were made with obtained stresses from the probabilistic approach (Harr 1977). According to the authors the particulate-probabilistic theory seems approximately correct when $K=K_{a}$.

As we can observe, uncertainties are inherent to the testing method, in other terms using interval values can be suitable to handle the perturbations. Intervals are often used as a tool to handle uncertainties which arise during experiments. In different types of tests uncertainties propagate during measuring procedures and from the use of different devices. Using Jaky's Formula for example uncertainty lies essentially in measuring the material angle of friction $\varphi^{\prime}$. Whatever the techniques used for measuring such a parameter, there are uncertainties to consider.

\section{FORMULATION OF INTERVAL-BASED PARAMETERS FOR DIFFUSION}

Interval analysis was introduced by Moore (1966) and is considered as a mathematical discipline that deals with quantities expressed as intervals which are common in engineering problems. Intervals are a convenient tool to deal with uncertainty when there is lack of data. As probabilistic approaches require important amounts of data, when in geotechnics information is scarce and small data available in general, the use of interval analysis could be of interest. In practice, it may be difficult sometime to get a large number of experimental data so we need an alternative method in which we may handle the uncertainty with few experimental data. In our problem, the diffusion parameter $\mathrm{D}$ will be considered as interval-based. Applied pressure on the ground can also be considered as an uncertain parameter expressed in terms of interval.

Finite difference schemes are usually applied to solve the type of diffusion equation we have in our case. We can either use a forward or backward scheme to solve it when dealing with deterministic values of the parameter $\mathrm{D}$.

$\frac{\partial \bar{S}_{z}}{\partial z}=D \frac{\partial^{2} \bar{S}_{z}}{\partial x^{2}}$

where

$$
D=K . z
$$

( For simplicity we use $S$ instead of $\bar{S}_{z}$ )

In finite difference method, the forward scheme can be expressed by:

$S_{j}^{i+1}=S_{j}^{i}+K z^{i} \frac{\Delta z}{(\Delta x)^{2}}\left(S_{j+1}^{i}-2 S_{j}^{i}+S_{j-1}^{i}\right)$

Index $i$ is used in $z$ direction and $j$ in $x$ direction.

The formulation of the problem to deal with interval valued parameters is conducted following interval arithmetic and rules. In Moore et al. (2009), Hanss (2005) we can find more details on derivation and application of interval arithmetic.

\subsection{Reminder on intervals}

Interval valued approach gained significant use in engineering especially when information is uncer-

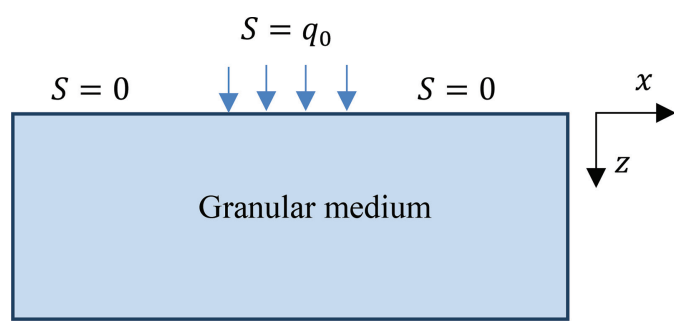

Figure 4. Scheme and Boundary Conditions. 
tain. Studies were conducted in different fields, such as thermal conductivity to account for uncertainty using interval approaches and fuzzy sets (Wang 2014). Wang (2014) proposed a new numerical technique named as fuzzy finite difference method to solve the heat conduction problems with fuzzy uncertainties in both the physical parameters and initial/boundary conditions. The $\alpha$ level-cut method is used to study the problem in terms of interval equations. Kermani \& Saburi (2007) presented a numerical method for solving "Fuzzy Partial Differential Equation" (FPDE) with some examples.

In soil mechanics the solutions for stress diffusion are based on continuum mechanics which considers the media as a continuum. Even constituted of particles, the granular aspect of the soil is not considered. The probabilistic approach from Harr (1977) considers the soil as a particulate media. The diffusion equation (1) is used with a parameter of diffusion $\mathrm{D}$ as a deterministic value. Deterministic values of $\mathrm{D}$ are not common, but we tend to use approximations. In the following the equation of diffusion of stresses in a granular medium will be considered in terms of intervals. The parameter of diffusion D and initial/boundary conditions are taken as intervals to account for uncertainty.

Using $\tilde{S}$, equation (3) can be written in terms of intervals as follows;

$$
\frac{\partial \tilde{S}}{\partial z}=\tilde{D} \frac{\partial^{2} \tilde{S}}{\partial x^{2}}
$$

$\tilde{S}$ is replaced by $S_{\alpha}^{I}$. We notice $S_{\alpha}^{I}$ for a given interval $I$ of $\alpha$ level. $\underline{S}(\alpha), \bar{S}(\alpha)$ are the lower and upper values of the interval at $\alpha$ level

$$
\begin{gathered}
\frac{\partial \tilde{S}}{\partial z}=\frac{\partial[\underline{S}(\alpha), \bar{S}(\alpha)]}{\partial z} \\
\frac{\partial S_{\alpha}^{I}}{\partial z}=\frac{S_{\alpha}^{I . i+1}-S_{\alpha}^{I . i}}{\Delta z} \\
\frac{\partial^{2} \tilde{S}}{\partial x^{2}}=\frac{\partial^{2} S_{\alpha}^{I}}{\partial x^{2}}=\frac{S_{\alpha, j+1}^{I}-2 S_{\alpha, j}^{I}+S_{\alpha, j-1}^{I}}{(\Delta x)^{2}}
\end{gathered}
$$

Then the forward scheme of finite difference approach using interval-based parameters can be written;

$S_{\alpha, j}^{I, i+1}=S_{\alpha, j}^{I, i}+D_{\alpha, j}^{I, i} \frac{\Delta z}{(\Delta x)^{2}}\left[S_{\alpha, j+1}^{I, i}-2 S_{\alpha, j}^{I, i}+S_{\alpha, j-1}^{I, i}\right](8)$

where

$$
D_{\alpha, j}^{I, i}=K_{\alpha, j}^{I, i} z_{j}^{i}
$$

$\alpha$ level cuts are used to characterize a fuzzy number.

A triangular fuzzy number, denoted by $\mathrm{u}=\langle\mathrm{a}, \mathrm{b}, \mathrm{c}\rangle$ where $\mathrm{a} \leq \mathrm{b} \leq \mathrm{c}$ has $\alpha$-cuts

$$
[\mathrm{u}]_{\alpha}=[\mathrm{a}+\alpha(\mathrm{b}-\mathrm{a}), \mathrm{c}-\alpha(\mathrm{c}-\mathrm{b})], \alpha \in[0,1]
$$

And membership function

$$
\mu_{T r i}(x)=\left\{\begin{array}{cl}
\frac{x-a}{b-a} & \text { if } a \leq x \leq b \\
\frac{c-x}{c-b} & \text { if } b \leq x \leq c \\
0 & \text { otherwise }
\end{array}\right.
$$

Elementary operations of interval arithmetic are used (Hanss, 2005).

$$
\begin{aligned}
& {\left[a_{1}, b_{1}\right]+\left[a_{2}, b_{2}\right]=\left[a_{1}+a_{2}, b_{1}+b_{2}\right]} \\
& {\left[a_{1}, b_{1}\right]-\left[a_{2}, b_{2}\right]=\left[a_{1}-b_{2}, b_{1}-a_{2}\right]} \\
& {\left[a_{1}, b_{1}\right] \times\left[a_{2}, b_{2}\right]=[\min (M), \max (M)]} \\
& M=\left\{a_{1} a_{2}, a_{1} b_{2}, b_{1} a_{2}, b_{1} b_{2}\right\}
\end{aligned}
$$

\section{CASE STUDY}

Turedi and Ornek (2017) performed laboratory experiment on sand to investigate the stress and bearing capacity. The vertical stresses resulting from strip and rectangular footings are measured at different depths of the tank model of dimensions $1.25 \mathrm{~m}$ length, $1.0 \mathrm{~m}$ width and $1.0 \mathrm{~m}$ depth. The loading is considered under a model footing of $\mathrm{B}=0.1 \mathrm{~m}$ breadth and $\mathrm{L}=5 \mathrm{~B}$ length. The measured average peak friction angles $\phi^{\prime}$ were $36^{\circ}$ and $42^{\circ}$ for loose and dense sands, respectively. The results of measured vertical stress $(\mathrm{kPa})$ are given in Figure 5.

The model was calibrated using the results from Turedi and Ornek (2017). Figure 6 shows the vertical stress distribution using the probabilistic approach when we consider a deterministic diffusion parameter $D=K . z$. Jaky's formula is used, $K=1-\sin \varphi^{\prime}$. As the experiment was performed in loose sand we used for our simulation $\varphi^{\prime}=36^{\circ}$ as given by the authors.

The purpose of our study is to take into account uncertainties using interval-valued parameters. The proposed model permits to consider the parameter of diffusion $\mathrm{D}$ in terms of intervals, or as triangular fuzzy number. The loading on the surface can be taken into account as interval also. 


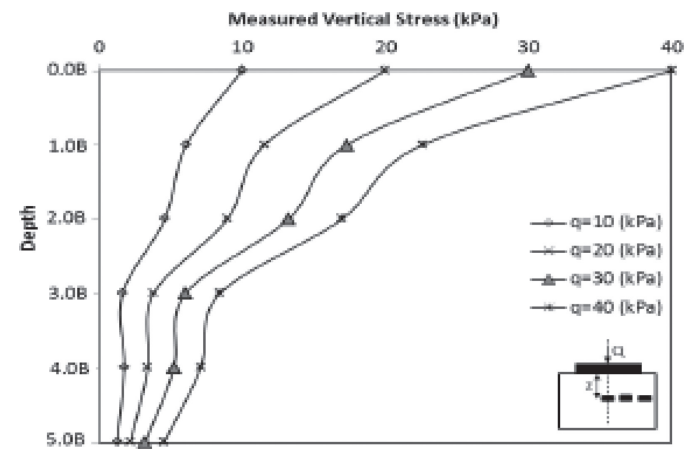

Figure 5. Vertical stress distribution along the depth at different loading levels (Experiment Turedi \& Ornek, 2017).

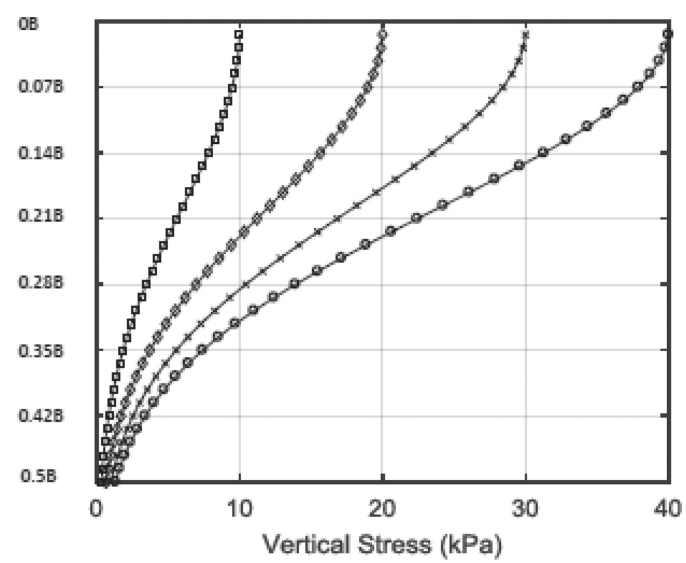

Figure 6. Vertical stress distribution along the depth at different loading levels (Probabilistic approach, $K=1$ $\left.-\sin \varphi^{\prime}\right)$.

Considering ranges of values for the coefficient of lateral pressure $K$ around the friction angle of sand $\varphi^{\prime}$, the used interval values of $K$ are given at different $\alpha$ level cuts. The applied load is taken as point valued $\mathrm{q}=40 \mathrm{kPa}$. The triangular fuzzy number for $K$ can be written in terms of intervals at level $\alpha,[K]_{\alpha}=[0.35+0.03 \alpha, 0.41-0.03 \alpha]$.

And the membership function is given as

$$
\mu_{K}(x)= \begin{cases}\frac{x-0.35}{0.03} & \text { if } 0.35 \leq x \leq 0.38 \\ \frac{0.41-x}{0.03} & \text { if } 0.38 \leq x \leq 0.41 \\ 0 & \text { otherwise }\end{cases}
$$

Figure 7 shows distribution of vertical stress at $\alpha$ cut levels; $\alpha 0=0, \alpha 1=1 / 3, \alpha 2=2 / 3$ and $\alpha 3=1$.

Parameter D being dependent on depth, it is noticed that uncertainty is more pronounced with $\mathrm{z}$ increasing. Uncertain parameter of diffusion has important impact on stress distribution, especially at deeper levels. The observed dispersion suggests that using deterministic values for D gives only an approximation of the stress. It can underestimate or overestimate the real level of stress in the medium. Using interval values for the diffusion parameter helps estimating the evolution of stress spreading with depth.

One can also consider uncertainty in the loading using intervals for the surficial charge q. Influence of interval values of $\mathrm{q}$ is shown in Figure 8 for $\mathrm{q}=[q, \bar{q}]=[38 \mathrm{kPa}, 40 \mathrm{kPa}]$. The parameter of diffusion is kept point valued in this case with $K=0.38$.

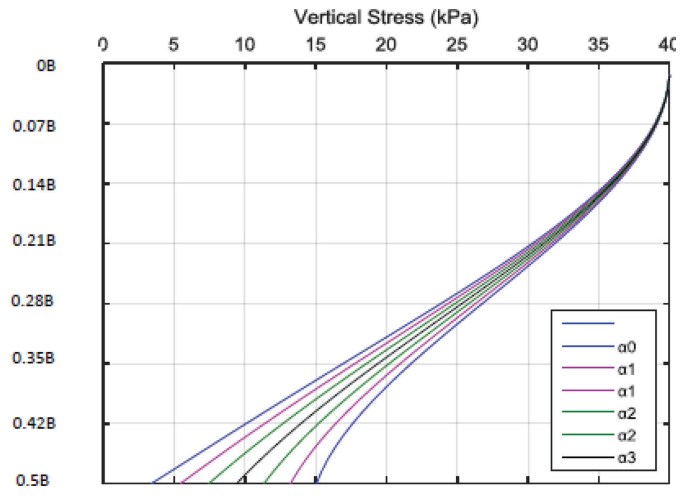

Figure 7. Vertical stress distribution under axis for different level cuts of $K$.

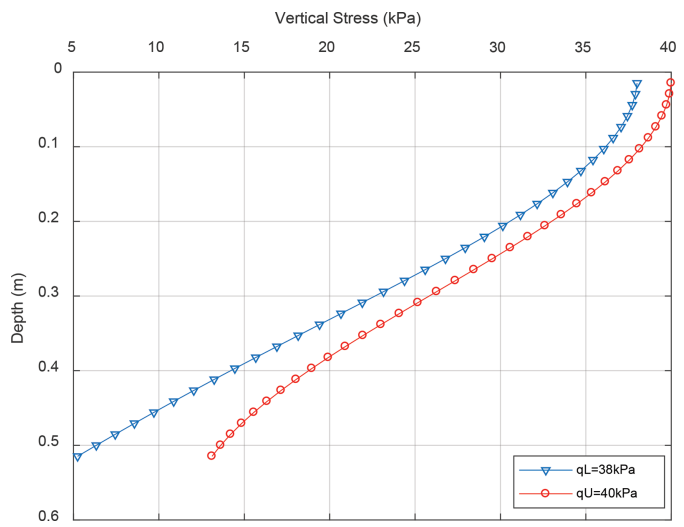

Figure 8. Vertical stress distribution under axis for inter-val surficial pressure $[q, \bar{q}]$. 


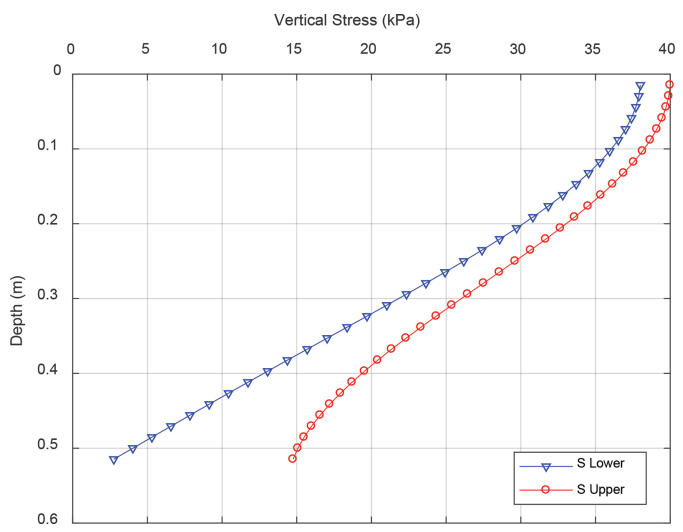

Figure 9. Vertical stress distribution for inter-val surficial pressure $[q, \bar{q}]$ and interval coefficient $[\underline{K}, \bar{K}]$.

The uncertainty on the load has effect on the distribution as the diffusion shows the vertical stress evolving as an interval from the surface, and it continues with the depth. It is slightly augmented for $z$ deeper than half of the domain.

Combination of uncertainty due to $K$ and q has higher effect on the evolution of uncertainty in vertical stress as it is illustrated in Figure 9. The surficial pressure is kept as $\mathrm{q}=[38 \mathrm{kPa}, 40 \mathrm{kPa}]$ and the lateral pressure coefficient $K=[0.35,0.41]$.

As uncertainty is combined between the surficial pressure $q$ and the coefficient $K$ we notice fast dispersion in the vertical stress $\mathrm{S}$ with depth as it was illustrated in Figure 7 already, but the effect is more pronounced because uncertainty originates from both the load and the soil parameter.

\section{CONCLUSION}

The probabilistic approach from Harr (1977) needed only one parameter for characterizing the particulate medium. It was shown in (Bourdeau, 1986) that lateral pressure coefficient can be a good estimate of the parameter for vertical stress diffusion in a granular soil. We revisited the theory from Harr to account for uncertainty in the diffusion parameter $\mathrm{D}$ and in the surficial loading. The purpose was to show the ability of interval valued method to handle uncertainty due to lack of knowledge and data. The finite difference scheme is practical for this type of equations. The construction of such a scheme for interval analysis is not straight forward as it obeys number of specific conditions. It was shown that uncertainty in the diffusion parameter affects significantly the distribution of vertical stress in the granular medium. And, when combined with the uncertainty from surficial pressure the stress distribution shows more dispersion with depth. Interval valued parameters are suitable when dealing with lack of data and uncertainty.

\section{REFERENCES}

Baecher GB. \& Christian JT. 2003. Reliability and Statistics in Geotechnical Engineering. Wiley.

Bourdeau PL. 1986. Analyse probabiliste des tassements d'un massif de sol granulaire. These de Doctorat es Sciences Techniques, No. 628, Swiss Federal Institute of Technology.

Cai G, Liu S, Puppala AJ, Tong L. 2011. Assessment of the coefficient of lateral earth pressure at rest $(\mathrm{K} 0)$ from in situ seismic tests. Geotech Test J 2011;34(4):1-11.

Chen TJ \& YS Fang. 2008. Earth Pressure due to Vibratory Compaction. Journal of Geotechnical and Geoenvironmental Engineering. 2008.134:437-444.

Hanss M.2005. Applied Fuzzy Arithmetic, An Introduction with Engineering Applications. Springer Verlag.

Harr ME. 1977. Mechanics of particulate media: A probabilistic approach. McGraw-Hill.

Kermani MA \& F. Saburi. 2007. Numerical Method for Fuzzy Partial Differential Equations. Applied Mathematical Sciences, Vol. 1, 2007, no. 27, 1299-1309.

Moore, RB Kearfott \& MJ Cloud. 2009. Introduction to interval analysis. Society for Industrial and Applied Mathematics SIAM, Philadelphia.

Nayak S. \& S. Chakraverty. 2015. Numerical solution of moving plate problem with uncertain parameters. arXiv:1503.07809v1.

Schmertmann JH. 2005. Stress Diffusion in Sand. Journal of Geotechnical and Geoenvironmental Engineering. Vol.131, No1, January 2005.

Turedi Y. \& M. Ornek. 2017. Stress Analyses of Strip and Rectangular Footings Rested on Loose Sands. NESciences, 2017, 2 (3): 93-112.

Wang C. \& Qiu ZP. 2014. Fuzzy finite difference method for heat conduction analysis with uncertain parameters. Acta Mechanica Sinica (2014) 30(3):383-390. DOI 10.1007/s10409-014-0036-7.

Wang C. \& Qiu ZP. 2014a. Interval finite difference method for steady-state temperature field prediction with interval parameters. Acta Mechanica Sinica (2014) 30(2):161-166. DOI 10.1007/s10409-0140020-2.

Zureigat HH \& AM Ismail. 2016. Numerical Solution of Fuzzy Heat Equation with Two Different Fuzzifications. SAI Computing Conference 2016.July 13-15, 2016. London, UK. 